\title{
Clinical Trial Team Training Document
}

National Cancer Institute

\section{Source}

National Cancer Institute. Clinical Trial Team Training Document. NCI Thesaurus. Code C125425.

Documentation attesting to completion of trial team training by the clinical trial sponsor (the pharmaceutical or biotech company). 